\title{
NEW OPTICAL OBSERVING SYSTEM IN SERIES ELECTRON BEAM GUNS
}

\author{
V.A. KRAMARENKO, V.M. NESTERENKOV and V.I. ZAGORNIKOV \\ E.O. Paton Electric Welding Institute, NASU \\ 11 Kazimir Malevich Str., 03680, Kiev, Ukraine. E-mail: office@paton.kiev.ua
}

\begin{abstract}
Different approaches were studied for welded butt observing in the vacuum chambers under conditions of intensive deposition. The results of development of new optical system suitable for application in industrially applied guns of ELA 60/60 type are given. The system differs by simplicity and possibility of wide application of standard assemblies. The system will find commercial application due to completeness of set, high functionality and simplicity. 3 Ref., 6 Figures.
\end{abstract}

Keywords : electron beam welding, electron beam gun, welded butt observing, optical system, optics protection, difficult-to-reach welding places

A process of EBW takes place at large rates that requires high accuracy of alignment of electron beam and butt being welded. The latter is assembled with a gap close to zero. An operator is located out of a vacuum chamber at some distance from a place of welding, therefore the issues of beam-to-butt combining, alignment of mutual location of beam and part are very relevant for the whole technological process $[1,2]$.

Currently, three types of view systems are developed in the commercial EB systems, i.e. viewports, optical and TV systems or their combinations. It is a well-known system RASTR [3] used for observing the welding place in secondary emission electrons and beam to butt alignment with $0.1 \mathrm{~mm}$ accuracy. A principle of RASTR equipment operation is based on measurement of current of the secondary emission electrons forming in cyclic (with $300 \mathrm{~ms}$ period) scanning of working zone of the part with low power fine-focused beam in short-term moments (to $5 \mathrm{~ms}$ ) of interruption of EBW process. An image of part surface is formed based on the signals from secondary emission electrons probe installed on the EB gun in the vicinity to welding place. Measured and digitized by observing equipment brightness levels in areas of scanned surface are recorded in computer memory in form of shot (matrix) of the image and displayed in the separate window of RASTR monitor after special software processing.

Low power fine-focused electron beams are used for welding of small-size precision parts, and the parts of 1-3 mm thickness are assembled with increased accuracy. Combined optical-TV systems with direct observing the welded butt through gun optical system can be useful for such parts.
This paper provides for the results of development of a new optical system suitable for application in widely applied in industry guns of ELA 60/60 type. Taking into account that the main drawback of the optical systems is deposition of mirrors with metal evaporating in welding, the work pays great attention to optics protection from deposition. It is a well-known fact that the protective devices based on stroboscopic effect are used under conditions of intensive deposition. Such systems are characterized by decrease of object illumination. It is possible to increase their efficiency by stabilizing rotation of a drum with up to $1 \mathrm{~Hz}$ accuracy on frequencies divisible by shot frequency $(50,1250,2500 \mathrm{~Hz}$ etc.) that is highly sophisticated.

Tape transporting mechanisms, changeable glasses, including protective rotating and shifting glasses [1] are widely used. They shall have compact structure, work in wide range of operating distances with sufficient supply of protective tape, which should not be deformed and stuck. Application of such mechanisms is possible only at sufficient distance from the beam. Removal of the optics from the electron beam results in significant distortions of the image on video camera, elimination of which requires thorough production (polishing of mirrors etc.) and complication of structure of view device, that resulted in refusal from this direction in development of the observing systems.

Proposed system differs from the earlier developed by simplicity and high level of assembly unity. The whole package does not require drastic changes in the structure of EB gun (Figure 1). A view optical system is set in a body of gun anode block with ap- 


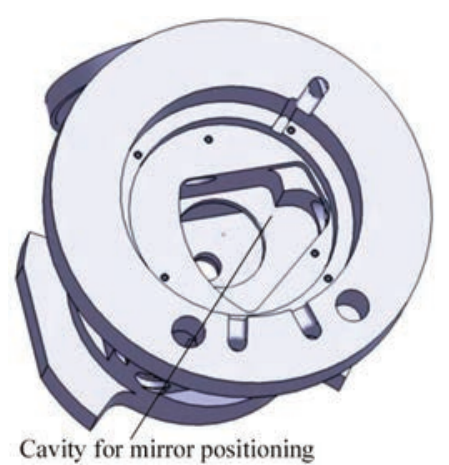

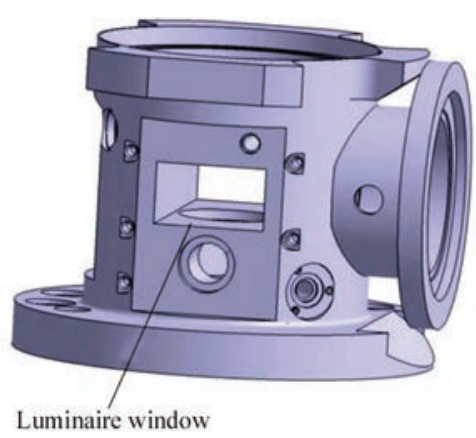

Figure 1. Improvement of body of anode block in EB gun ELA 60/60

proximated to the maximum coaxial alignment of the axis of electron beam to the optical system axis.

The body has a milled-out cavity for positioning mirror mechanism and a hole for axis of external electric motor of mirrors drive. Few threaded holes were added outside of the body for fixing mounted blocks of video monitoring unit. Mirror mechanisms board 1 has axis 3 relatively to which mirror holder 2 is rotated (Figure 2). In welding mode the mirrors are removed from a beam channel of the gun and do not effect the welding parameters. The image is absent in this case and access of mirror-deposition vapours from the materials being welded is blocked. In a setup mode the mirrors are positioned across the optical axis of luminaire 6 and objective lens 7 by means of pinion shaft 5 of electric drive and gear sector 4 of mirror holder 2. Light from the luminaire is directed by own mirror on part section being welded and via reflection from another mirror is directed along the optical axis of objective lens and CCD-camera. Mirror holder design prevents direct entering of the luminaire beams in CCD-camera objective lens.

This mode assumes turning on of electron beam with $0.8-1.5 \mathrm{~mA}$ current intensity, which flows through the hole between the mirrors and allows observing brightened point in a place of beam entering into billet being welded. Deposition of the mirrors was not virtually observed at such a current. In order to prevent mirrors' deposition the program for welding mode regulation blocks beam current rise, if the mirrors are not removed from the beam channel. Miniature electric drive 3 (see Figure 2) with rotating moment of around $10 \mathrm{~kg} / \mathrm{cm}$ is used for mirror holder rotation. Requirements on absence of magnetic field in inactive mode and good heat-sink in vacuum are made to it. Besides, scheme of its regulation should stipulate current limitation at 120-150\% level from nominal at mechanism stopping in lock and further power shut down. The video system is made in from of removable unit, in which objective lenses can be replaced depending on set requirements. The objective lenses have an electro-mechanical drive for regulation of focus and diaphragm, that allows easy adjustment of image quality. The CCD-camera itself is located in a hermetic enclosure (Figure 3 ) which is mounted to objective lens body with the help of coupling nut. Air in a CCD-camera box provides for sufficient heat exchange of electronic circuit with body. Further, heat is dissipated over the parts of objective lens body and EB gun.

Figures 4 and 5 show the video-monitoring unit in section. Observing position of the mirrors opens the channels in the mirror holder for luminous flux from luminaire to objective lens. The luminous flux of source (white arrows) comes to mirror 1 and being directed to welding zone area. The image of this zone (grey arrows) is reflected from mirror 2 and guided to the objective lens. The angles of mirror positioning are set and calculated for showing the welding zone in $50-500 \mathrm{~mm}$ range from EB gun edge. Flow of electrons from the cathode passes through the hole between mirrors of $5 \mathrm{~mm}$ diameter and in this case a

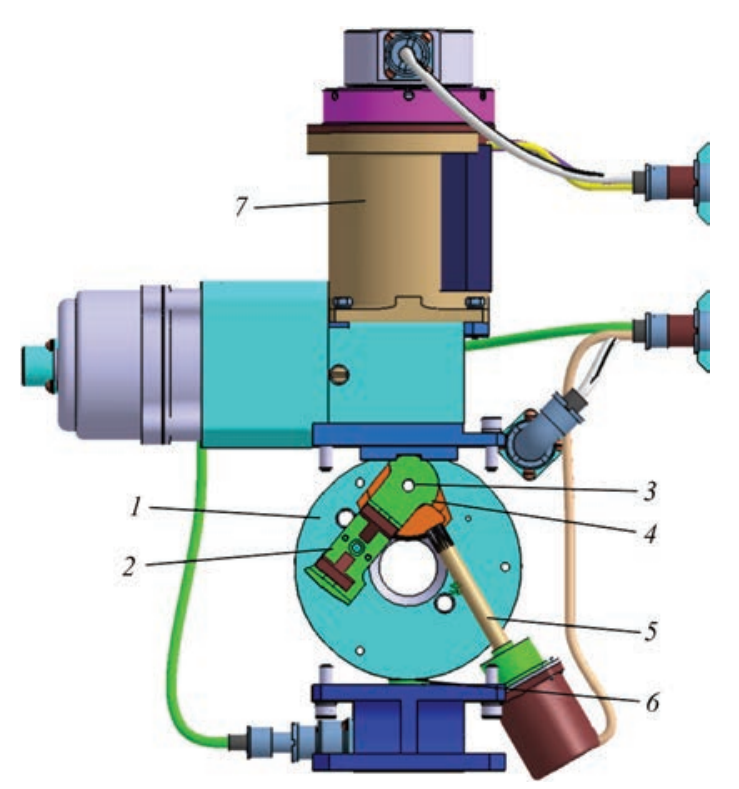

Figure 2. View of mechanism of mirror drive (see designations in the text) 


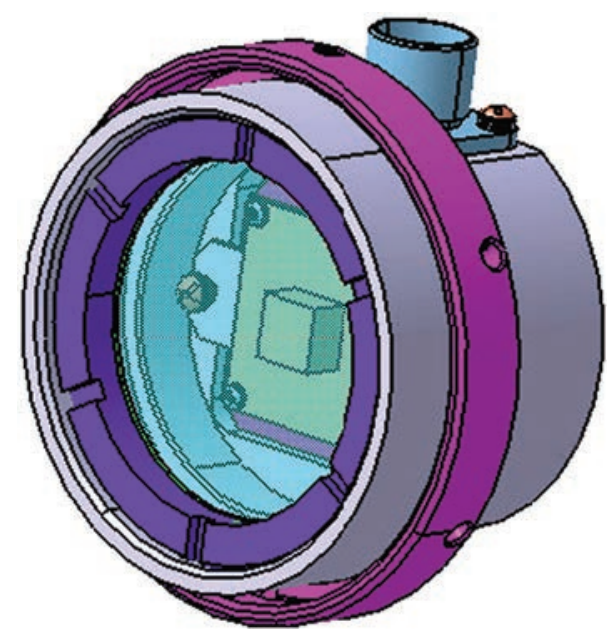

Figure 3. View of CCD-camera body

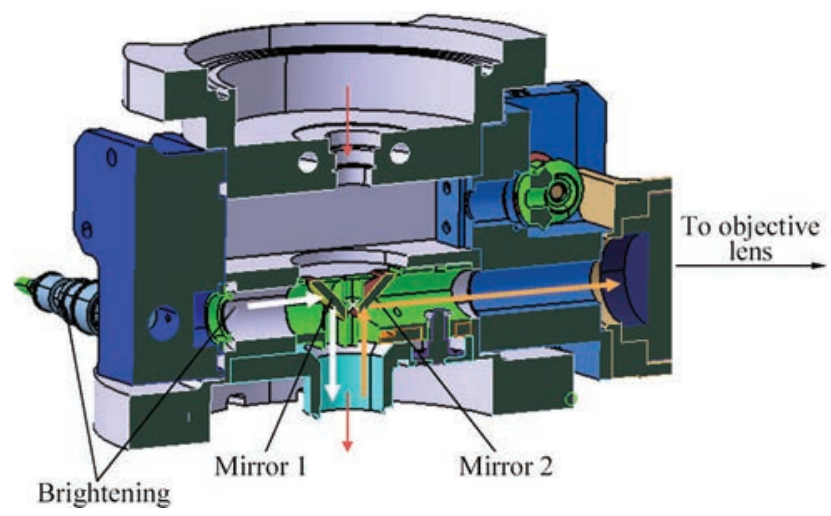

Figure 4. Cross section of optical observing system

brightened point of contact of beam with metal surface can be observed in the image.

Before welding the mirrors are removed sideways from the beam channel (mirror holder is turned to $40^{\circ}$ ). At that for preventing metal evaporation the objective lens is closed by half-round backside of the mirror holder, and a special pivoting spring-loaded shutter is used for lens of the luminaire. Two light emitting diodes indicate position on control panel, namely red - beam channel is closed, beam current is limited by $0.8-1.2 \mathrm{~mA}$ value, and green - mirrors are removed, the system is ready for welding on operating current.

Two V-positioned mirrors are located under gun beam deflector in the proposed device. One of these mirrors directs the light beam from luminaire to part being welded, and another directs the light reflected from the surface of part with butt being welded (see Figure 4) into eyelens. Polished copper plates were used as mirrors since application of glass mirrors is complicated due to formation on glass of a static electron discharge, distorting electron beam, as well as flares deteriorating visibility.

A running block of mirrors mounted on an arm (Figure 6) is located on a flange embedded in the gun

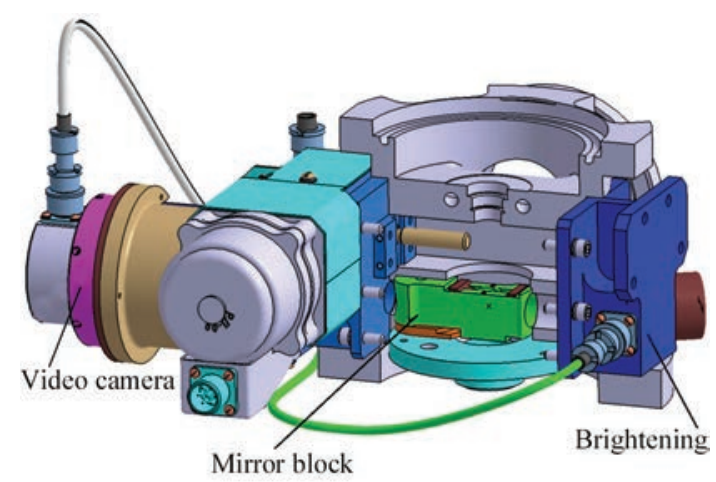

Figure 5. View of observing system with mirror rotation mechanism

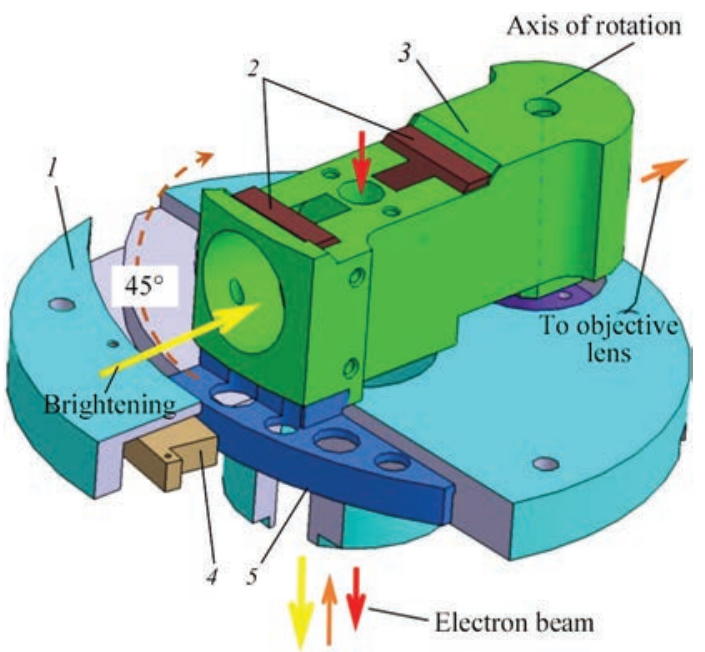

Figure 6. View of running mirror block: 1 - video block body; 2 - mirrors, 3 - rotating body of mirrors; 4 - piezocartridge; 5 - piezodrive surface

body. Balance wheel of the block, having radial movement with the help of reversible piezomotor, allows quick switch from tracking to welding mode. Advantage of the piezodrive lies in absence of magnetic fields during its operation. Photolens and TV-camera, image from which is displayed on the computer monitor, provide for five-fold magnification of the image at controlled area to $100 \mathrm{~mm}^{2}$ depending on operating distance.

Proposed observing system allowed realizing welding in difficult-to-reach places, working through EBW procedure for critical thin-walled parts with circumferential welds, some of which are deepened in the part body to $200 \mathrm{~mm}$.

1. Chvertko, A.I., Nazarenko, O.K., Svyatsky, A.M. et al. (1973) Equipment for electron beam welding. Kiev: Naukova Dumka.

2. Schiller, S., Heisig, U., Panzer, S. (1980) Electron beam technology. Moscow: Energiya.

3. Nazarenko, O.K., Shapoval, V.I., Loskutov, G.A. et al. (1993) Supervision of electron beam welding process and automatic joint tracking. Avtomatich. Svarka, 5, 35-38. 